\title{
BioMedicine
}

\section{Hyperuricemia might be an early manifestation of undiagnosed adult leukemia in a population-based cohort study}

Follow this and additional works at: https://www.biomedicinej.com/biomedicine

Part of the Life Sciences Commons, and the Medical Sciences Commons (c) (i)

This work is licensed under a Creative Commons Attribution 4.0 License.

\section{Recommended Citation}

Lai, Shih-Wei; Lin, Cheng-Li; and Liao, Kuan-Fu (2020) "Hyperuricemia might be an early manifestation of undiagnosed adult leukemia in a population-based cohort study," BioMedicine: Vol. 10 : Iss. 1 , Article 5. DOI: $10.37796 / 2211-8039.1004$

This Original Articles is brought to you for free and open access by BioMedicine. It has been accepted for inclusion in BioMedicine by an authorized editor of BioMedicine. 


\title{
Hyperuricemia might be an early manifestation of undiagnosed adult leukemia in a population-based cohort study
}

\author{
Shih-Wei Lai ${ }^{\mathrm{a}, \mathrm{b}}$, Cheng-Li Lin ${ }^{\mathrm{a}, \mathrm{c}}$, Kuan-Fu Liao ${ }^{\mathrm{d}, \mathrm{e}, *}$ \\ ${ }^{\text {a }}$ College of Medicine, China Medical University, Taichung, Taiwan \\ ${ }^{\mathrm{b}}$ Department of Family Medicine, China Medical University Hospital, Taichung, Taiwan \\ ${ }^{\mathrm{c}}$ Management Office for Health Data, China Medical University Hospital, Taichung, Taiwan \\ ${ }^{\mathrm{d}}$ College of Medicine, Tzu Chi University, Hualien, Taiwan \\ ${ }^{\mathrm{e}}$ Division of Hepatogastroenterology, Department of Internal Medicine, Taichung Tzu Chi General Hospital, Taichung, Taiwan
}

\begin{abstract}
Background/objective: No published population-based study investigates the association between hyperuricemia and undiagnosed adult leukemia in Taiwan. The aim of the study was to investigate whether hyperuricemia might be an early manifestation of undiagnosed adult leukemia in Taiwan.

Methods: A population-based cohort study was conducted to analyze the database of the Taiwan National Health Insurance Program. There were 47708 subjects aged 20 to 84 years with newly diagnosed hyperuricemia as the hyperuricemia group from 2000 to 2013, and 190832 randomly selected subjects without hyperuricemia as the non-hyperuricemia group. The hyperuricemia group and the non-hyperuricemia group were followed for one year to estimate the incidence of new diagnosis of leukemia.

Results: The overall incidence of leukemia was 1.32-fold higher in the hyperuricemia group than the non-hyperuricemia group (0.74 versus 0.55 per 10000 person-months, $95 \%$ confidence interval $1.28-1.37)$. The incidence rate ratio of leukemia was statistically higher in the first 3 months of hyperuricemia diagnosis (incidence rate ratio 4.05).

Conclusion: Adults with hyperuricemia have a higher incidence of being diagnosed with leukemia than those without hyperuricemia. Hyperuricemia might be an early manifestation of undiagnosed adult leukemia. Clinicians should check the complete blood count with differential to detect the possibility of leukemia when adults present with hyperuricemia.
\end{abstract}

Keywords: adult, hyperuricemia, leukemia, Taiwan National Health Insurance Program

\section{Introduction}

$\mathrm{L}$ eukemia is a cancer of blood, arising from blood cell types. Leukemia was as the ninth commonest cancer in the world in 2016 [1]. There were about 467000 new cases of leukemia, which accounted for $2.71 \%$ of 17228000 new cancer cases in the world in 2016 [1]. Leukemia ranked the thirteenth leading cause of cancer death in Taiwan in 2018 [2]. There were 1096 deaths due to leukemia, which accounted for $2.25 \%$ of 48784 cancer deaths in Taiwan in 2018 [2].

Early manifestations of leukemia before diagnosis might be vague or non-specific, including fatigue, fever, pallor, bleeding tendency, body weight loss, night sweats, insomnia, loss of appetite, ophthalmic involvement, gingival enlargement, and others [3-13]. When leukemia is treated with chemotherapy, hyperuricemia might develop due to rapid turnover of tumor cells [14-25]. Yet, no systemic analysis focuses on the association 
between hyperuricemia and undiagnosed adult leukemia.

The prevalence of hyperuricemia was high in the general population [26-31], but little is known regarding the association hyperuricemia and undiagnosed adult leukemia in Taiwan. If hyperuricemia is an early manifestation of undiagnosed adult leukemia, these undiagnosed leukemia patients might have a chance to be early detected and they might have a chance to be early treated. Therefore, a population-based cohort study was conducted to investigate this issue.

\section{Materials and methods}

\subsection{Study design and data source}

A population-based cohort study was conducted to analyze the database of the Taiwan National Health Insurance Program. The program was launched in March 1, 1995, and it has covered about $99.7 \%$ of 23 million people living in Taiwan [32, 33]. The database included sex, date of birth, and information on ambulatory care, hospitalization care, dental care, emergency care, and prescribed medications [34-40].

\subsection{Selection of subjects}

Subjects aged 20 to 84 years with newly diagnosed hyperuricemia and/or with a new attack of gout from 2000 to 2013 were selected as the hyperuricemia group (the International Classification of Diseases 9th Revision, ICD-9 codes 790.6 and 274). For each subject in the hyperuricemia group, approximately 4 subjects without hyperuricemia and gout were randomly selected as the non-hyperuricemia group. The index date was defined as the date of subjects being diagnosed with hyperuricemia or gout. The hyperuricemia group and the non- hyperuricemia group were matched with sex, age (every 5-year interval), and the year of index date. Subjects with a history of any cancer before the index date were excluded from the study.

\subsection{Major outcome}

The major outcome was a new diagnosis of leukemia (ICD-9 codes 204-208) during the follow-up period. All study subjects were followed for one year.

\subsection{Statistical analysis}

We compared the differences of sex and age between the hyperuricemia group and the non-hyperuricemia group by the Chi-square test for categorized variables and the $t$-test for continuous variables. The incidence of leukemia was estimated as the event number of leukemia detected during the follow-up period, divided by the total follow-up person-months for each group. The incidences of leukemia stratified by sex, age, and follow-up period between the hyperuricemia group and the non-hyperuricemia group were estimated. The incidence rate ratio (IRR) and the $95 \%$ confidence interval (CI) of leukemia in the hyperuricemia group versus the non-hyperuricemia group were estimated. All analyses were performed by the SAS 9.2 version (SAS Institute Inc., Carey, North Carolina, USA). The results were considered statistically significant when two-tailed $P$ values were $<0.05$.

\section{Results}

\subsection{Baseline information of the study population}

Table 1 reveals the baseline information of the study population. There were 47708 subjects in the hyperuricemia group and 190832 subjects in the

Table 1. Baseline characteristics of the study population.

\begin{tabular}{|c|c|c|c|c|c|}
\hline \multirow[t]{2}{*}{ Variable } & \multicolumn{2}{|c|}{$\begin{array}{l}\text { Non-hyperuricemia } \\
\mathrm{N}=190832\end{array}$} & \multicolumn{2}{|c|}{ Hyperuricemia $\mathrm{N}=47708$} & \multirow[t]{2}{*}{$P$ value $^{\mathrm{a}}$} \\
\hline & $\mathrm{n}$ & $(\%)$ & $\mathrm{n}$ & $(\%)$ & \\
\hline \multicolumn{5}{|l|}{ Sex } & \multirow[t]{3}{*}{0.001} \\
\hline Female & 61014 & $(32.0)$ & 16154 & (33.9) & \\
\hline Male & 129818 & $(68.0)$ & 31554 & $(66.1)$ & \\
\hline \multicolumn{5}{|l|}{ Age group (years) } & \multirow{4}{*}{0.001} \\
\hline $20-39$ & 61049 & $(32.0)$ & 15497 & $(32.5)$ & \\
\hline $40-64$ & 97551 & $(51.1)$ & 23791 & $(49.9)$ & \\
\hline $65-84$ & 32232 & $(16.9)$ & 8420 & $(17.6)$ & \\
\hline $\begin{array}{l}\text { Age (years), mean } \pm \text { standard } \\
\text { deviation }^{\mathrm{b}}\end{array}$ & \multicolumn{2}{|c|}{$48.6 \pm 15.4$} & \multicolumn{2}{|c|}{$48.6 \pm 15.5$} & 0.27 \\
\hline
\end{tabular}

Data are presented as the number of subjects in each group with percentages given in parentheses.

${ }^{a}$ Chi-square test and.

b $t$-test comparing the hyperuricemia group and the non-hyperuricemia group. 
Table 2. Incidence of leukemia stratified by sex, age, and follow-up period between the hyperuricemia group and the non-hyperuricemia group.

\begin{tabular}{|c|c|c|c|c|c|c|c|c|c|c|}
\hline \multicolumn{5}{|c|}{ Non-hyperuricemia } & \multicolumn{6}{|c|}{ Hyperuricemia } \\
\hline Variable & $\mathrm{N}$ & Event & Person-months & Incidence $^{a}$ & $\bar{N}$ & Event & Person-months & Incidence $^{\mathrm{a}}$ & $\mathrm{IRR}^{\mathrm{b}}$ & $(95 \% \mathrm{CI})$ \\
\hline All & 190832 & 126 & 2276984 & 0.55 & 47708 & 42 & 571180 & 0.74 & 1.32 & $(1.28,1.37)$ \\
\hline \multicolumn{11}{|l|}{ Sex } \\
\hline Female & 61014 & 50 & 728563 & 0.69 & 16154 & 16 & 193388 & 0.83 & 1.19 & $(1.12,1.26)$ \\
\hline Male & 129818 & 76 & 1548421 & 0.49 & 31554 & 26 & 377791 & 0.69 & 1.42 & $(1.36,1.48)$ \\
\hline \multicolumn{11}{|c|}{ Age group (years) } \\
\hline $20-39$ & 61049 & 21 & 728860 & 0.29 & 15497 & 10 & 185809 & 0.54 & 1.88 & $(1.77,1.99)$ \\
\hline $40-64$ & 97551 & 68 & 1167195 & 0.58 & 23791 & 17 & 285081 & 0.60 & 1.04 & $(0.98,1.09)$ \\
\hline $65-84$ & 32232 & 37 & 380929 & 0.97 & 8420 & 15 & 100291 & 1.50 & 1.54 & $(1.42,1.66)$ \\
\hline \multicolumn{11}{|c|}{ Follow-up period (months) } \\
\hline$\leq 3$ & 190832 & 4 & 571701 & 0.07 & 47708 & 4 & 143077 & 0.28 & 4.05 & $(3.90,4.20)$ \\
\hline $3-12$ & 190306 & 122 & 1705283 & 0.72 & 47666 & 38 & 428103 & 0.89 & 1.23 & $(1.19,1.28)$ \\
\hline
\end{tabular}

a Incidence rate: per 10000 person-months.

b IRR (incidence rate ratio, adjusted for sex and age): hyperuricemia versus non-hyperuricemia ( $95 \%$ confidence interval).

non-hyperuricemia group. The mean ages (standard deviation) of the study subjects were 48.6 (15.5) years for the hyperuricemia group and 48.6 (15.4) years for the non-hyperuricemia group, without statistical significance ( $t$-test, $P=0.27$ ).

\subsection{Incidence of leukemia stratified by sex age, and follow-up period}

Table 2 reveals that after adjusted for sex and age, the overall incidence of leukemia was 1.32 -fold higher in the hyperuricemia group than that in the non-hyperuricemia group $(0.74$ versus 0.55 per 10000 person-months, 95\% CI 1.28-1.37). The incidences of leukemia, as stratified by sex, age, and follow-up period, were all statistically higher in the hyperuricemia group than those in the non-hyperuricemia group. Subjects aged 65 to 84 years in the hyperuricemia group had a particularly higher incidence of leukemia (1.5 per 10000 personmonths). The incidence rate ratio of leukemia was statistically higher in the first 3 months of follow-up (incidence 0.28 versus 0.07 per 10000 personmonths, incidence rate ratio $4.05,95 \%$ CI 3.90-4.20). The incidence rate ratio was reduced to 1.23 during 3-12 months of follow-up (incidence 0.89 versus 0.72 per 10000 person-months, 95\% CI 1.19-1.28).

\section{Discussion}

To the best of our knowledge, this is the first population-based study to investigate the association between hyperuricemia and undiagnosed adult leukemia in Taiwan. No other study can be compared with each other. In order to reduce the biased results in our study, subjects with a history of any cancer before the index date were excluded from the study. All study subjects were followed until leukemia was newly diagnosed or for one year. Therefore, leukemia patients in our study were new cases. The diagnosis of hyperuricemia in the study was really prior to the confirmed diagnosis of leukemia. Therefore, hyperuricemia is less likely to be secondary to chemotherapy for leukemia or other cancers.

Although not unexpected findings, we noted that the overall incidence of leukemia was 1.32 -fold higher in the hyperuricemia group than that in the non-hyperuricemia group. The incidence rate ratio of leukemia was statistically higher in the first 3 months (incidence rate ratio 4.05). Although the outcome number was small (only 42 leukemia patients), our findings highlight that hyperuricemia might be an early manifestation of undiagnosed adult leukemia, particularly within three months of hyperuricemia diagnosis.

We reviewed the relevant literature to explain the association between hyperuricemia and undiagnosed adult leukemia. Leukemia cells have a tendency of rapid turnover, which leads to rapid breakdown of these tumor cells and subsequent hyperuricemia [14-17, 22]. Because early manifestations of leukemia before confirmed diagnosis might be vague or non-specific, it is difficult to early diagnose leukemia. We suggest that clinicians should check the complete blood count with differential to detect the possibility of leukemia when adults present with hyperuricemia.

\section{Limitation}

Several limitations of the study should be discussed. First, due to the inherent limitation of the database, other manifestations of leukemia before confirmed diagnosis were not documented. We could not determine hyperuricemia or other manifestations caming first. During disease progression of different stages, leukemia patients would have different manifestations, but our study could not 
verify it. Second, due to the eligible number of leukemia patients being small, we could not make a comparative analysis between acute and chronic leukemia. We could only analyze the association between hyperuricemia and the overall leukemia. Moreover, it indicates a future research direction. Third, In view of the availability of the Taiwan's medical care, it is not necessary to spend one year to diagnose leukemia since the onset of leukemiarelated manifestations. That was why subjects in our study were only followed for one year.

We conclude that adults with hyperuricemia have a higher incidence of being diagnosed with leukemia than those without hyperuricemia. Hyperuricemia might be an early manifestation of undiagnosed adult leukemia. Clinicians should check the complete blood count with differential to detect the possibility of leukemia when adults present with hyperuricemia.

\section{Specific author contributions}

Shih-Wei Lai contributed to the conception of the article, initiated the draft of the article, and has approved the final draft submitted.

Cheng-Li Lin and Kuan-Fu Liao conducted data analysis.

\section{Ethical statement}

Insurance reimbursement claims data used in this study were available for public access. Patient identification numbers were scrambled to ensure confidentiality. Patient informed consent was not required. This study was approved by the Research Ethics Committee of China Medical University and Hospital in Taiwan (CMUH-104-REC2-115).

\section{Acknowledgments}

This study was supported in part by the Ministry of Health and Welfare in Taiwan (MOHW109-TDUB-212-114004) and MOST Clinical Trial Consortium for Stroke (MOST 108-2321-B-039-003). These funding agencies did not influence the study design, data collection and analysis, decision to publish, or preparation of the manuscript.

\section{Conflicts of interest statement}

The authors disclose no conflicts of interest.

\section{References}

[1] Global, regional, and national incidence, prevalence, and years lived with disability for 328 diseases and injuries for 195 countries, 1990-2016: a systematic analysis for the
Global Burden of Disease Study 2016. Lancet 2017;390: 1211-59.

[2] Ministry of Health and Welfare Taiwan. statistics of causes of death. 2018 [cited on August 1, 2019, English version], http:// www.mohw.gov.tw/EN/Ministry/Index.aspx.

[3] Abbott BL. Chronic lymphocytic leukemia: recent advances in diagnosis and treatment. Oncologist 2006;11:21-30.

[4] Shahab F, Raziq F. Clinical presentations of acute leukemia. J Coll Physicians Surg Pak 2014;24:472-6.

[5] Shephard EA, Neal RD, Rose PW, Walter FM, Hamilton W. Symptoms of adult chronic and acute leukaemia before diagnosis: large primary care case-control studies using electronic records. Br J Gen Pract 2016;66:e182-8.

[6] Rose-Inman H, Kuehl D. Acute Leukemia. Hematol Oncol Clin North Am 2017;31:1011-28.

[7] Viele CS. Diagnosis, treatment, and nursing care of acute leukemia. Semin Oncol Nurs 2003;19:98-108.

[8] Devine SM, Larson RA. Acute leukemia in adults: recent developments in diagnosis and treatment. CA Cancer J Clin 1994;44:326-52.

[9] Leak Bryant A, Coffman EM, Phillips B, Gray TF, Knafl GJ, Klepin HD, et al. Symptoms, Mobility and Function, and Quality of Life in Adults With Acute Leukemia During Initial Hospitalization. Oncol Nurs Forum 2018;45:653-64.

[10] Leak Bryant A, Lee Walton A, Shaw-Kokot J, Mayer DK, Reeve BB. Patient-reported symptoms and quality of life in adults with acute leukemia: a systematic review. Oncol Nurs Forum 2015;42:E91-101.

[11] Albrecht TA. Physiologic and psychological symptoms experienced by adults with acute leukemia: an integrative literature review. Oncol Nurs Forum 2014;41:286-95.

[12] Hafeez MU, Ali MH, Najib N, Ayub MH, Shafi K, Munir M, et al. Ophthalmic Manifestations of Acute Leukemia. Cureus 2019;11.

[13] Lim HC, Kim CS. Oral signs of acute leukemia for early detection. J Periodontal Implant Sci 2014 Dec;44(6):293-9. https://doi.org/10.5051/jpis.2014.44.6.293. Epub 2014 Dec 31.

[14] Sevinir B, Demirkaya M, Baytan B, Gunes AM. Hyperuricemia and tumor lysis syndrome in children with nonHodgkin's lymphoma and acute lymphoblastic leukemia. Turk J Haematol 2011;28:52-9.

[15] Rajendran A, Bansal D, Marwaha RK, Singhi SC. Tumor lysis syndrome. Indian J Pediatr 2013;80:50-4.

[16] Naeem B, Moorani KN, Anjum M, Imam U. Tumor lysis syndrome in pediatric acute lymphoblastic leukemia at tertiary care center. Pak J Med Sci 2019;35:899-904.

[17] Tasmeen R, Islam A, Alam ST, Begum M. Tumor Lysis Syndrome and Hyperleukocytosis in Childhood Acute Lymphoblastic Leukemia in a Tertiary Care Hospital. Mymensingh Med J 2017;26:906-12.

[18] Gopakumar KG, Thankamony P, Seetharam S. Treatment of tumor lysis syndrome in children with leukemia/lymphoma in resource-limited settings-Efficacy of a fixed low-dose rasburicase. Pediatr Hematol Oncol 2017;34:206-11.

[19] Ribeiro RC, Pui CH. Recombinant urate oxidase for prevention of hyperuricemia and tumor lysis syndrome in lymphoid malignancies. Clin Lymphoma 2003;3:225-32.

[20] Cheson BD, Heitner Enschede S, Cerri E, Desai M, Potluri J, Lamanna N, et al. Tumor Lysis Syndrome in Chronic Lymphocytic Leukemia with Novel Targeted Agents. Oncologist 2017;22:1283-91.

[21] Okay M, Cetik S, Haznedaroglu IC. Tumor Lysis Syndrome Due to Targeting of Hepatocellular Carcinoma Associated with Chronic Myelomonocytic Leukemia. Turk J Haematol 2019;36(3):218-9.

[22] Firwana BM, Hasan R, Hasan N, Alahdab F, Alnahhas I, Hasan S, et al. Tumor lysis syndrome: a systematic review of case series and case reports. Postgrad Med 2012;124:92-101.

[23] Naithani R, Bhat A, Bhasin A. Bendamustine induced tumor lysis syndrome with acute renal failure in chronic lymphocytic leukemia. Indian J Cancer 2014;51:380-1.

[24] Montesinos P, Lorenzo I, Martin G, Sanz J, PerezSirvent ML, Martinez D, et al. Tumor lysis syndrome in 
patients with acute myeloid leukemia: identification of risk factors and development of a predictive model. Haematologica 2008;93:67-74.

[25] Koratala A. Tumor lysis syndrome with massive hyperphosphatemia and hyperuricemia. Clin Case Rep 2017;5: 2158-9.

[26] Lai SW, Liu CS, Lin T, Lin CC, Lai HC, Liao KF. Prevalence of gout and hyperuricemia in Taiwan: a hospital-based, cross-sectional study. South Med J 2009;102:772-3.

[27] Paul BJ, Anoopkumar K, Krishnan V. Asymptomatic hyperuricemia: is it time to intervene? Clin Rheumatol 2017;36: 2637-44.

[28] Cho SK, Winkler CA, Lee SJ, Chang Y, Ryu S. The Prevalence of Hyperuricemia Sharply Increases from the Late Menopausal Transition Stage in Middle-Aged Women. J Clin Med 2019;8:E296.

[29] Ali N, Perveen R, Rahman S, Mahmood S, Islam S, Haque T, et al. Prevalence of hyperuricemia and the relationship between serum uric acid and obesity: A study on Bangladeshi adults. PLoS One 2018;13. e0206850.

[30] Billa G, Dargad R, Mehta A. Prevalence of Hyperuricemia in Indian Subjects attending Hyperuricemia Screening Programs-A Retrospective Study. J Assoc Physicians India 2018; 66:43-6.

[31] Kim Y, Kang J, Kim GT. Prevalence of hyperuricemia and its associated factors in the general Korean population: an analysis of a population-based nationally representative sample. Clin Rheumatol 2018;37:2529-38.

[32] Ministry of Health and Welfare Taiwan. Taiwan Health and Welfare Report. 2018 [cited on August 1, 2019, English version], http://www.mohw.gov.tw.
[33] Liao KF, Kuo YH, Lai SW. Increased Risk of Herpes Zoster in Cancer Patients. J Infect Dis 2019;220:1542-3.

[34] Lin YS, Yeh CC, Huang SF, Chou YS, Kuo LT, Sung FC, et al. Aspirin associated with risk reduction of secondary primary cancer for patients with head and neck cancer: A populationbased analysis. PLoS One 2018;13. e0199014.

[35] Tzang RF, Chang YC, Kao KL, Huang YH, Huang HC, Wang YC, et al. Increased risk of developing psychiatric disorders in children with attention deficit and hyperactivity disorder (ADHD) receiving sensory integration therapy: a population-based cohort study. Eur Child Adolesc Psychiatry 2019;28:247-55.

[36] Chan PC, Chang WL, Hsu $\mathrm{MH}$, Yeh $\mathrm{CH}$, Muo $\mathrm{CH}$ Chang KS, et al. Higher stroke incidence in the patients with pancreatic cancer: A nation-based cohort study in Taiwan. Medicine 2018;97. e0133.

[37] Chou YL, Liou JT, Cheng CC, Tsai MC, Lin WS, Cheng SM, et al. The association of ischaemic stroke in patients with heart failure without atrial flutter/fibrillation. Heart 2019.

[38] Wang IK, Tsai TH, Hung YC, Wang TY, Yen TH, Lin CL, et al. Increased risk of new-onset type 2 diabetes in people with chronic kidney disease. Int Urol Nephrol 2019;51: 707-12.

[39] Ju UH, Liu FC, Lin CS, Huang WY, Lin TY, Shen CH, et al. Risk of Parkinson disease in Sjogren syndrome administered ineffective immunosuppressant therapies: A nationwide population-based study. Medicine 2019;98. e14984.

[40] Hung PH, Yeh CC, Sung FC, Hsiao CY, Muo CH, Hung KY, et al. Erythropoietin prevents dementia in hemodialysis patients: a nationwide population-based study. Aging 2019;11: 6941-50. 